Case Report

\section{Colonic tuberculosis}

\section{Introduction}

Tuberculosis is endemic in Tunisia. One-third of the world's population is infected with tuberculosis (TB), with intestinal TB representing the sixth most common presentation of extra-pulmonary TB. ${ }^{1}$ The diagnosis of intestinal TB is a challenge for physicians due to its diverse clinical manifestations that mimic other infectious, autoimmune, and tumor disorders, and is thus rarely considered as the causative agent of disease. Therefore, a high index of suspicion is essential to reach the correct diagnosis.

We report 3 cases of colonic tuberculosis which illustrate the variety of background circumstances and clinical manifestations that it may comprise.

\section{Cases presentation}

\section{Case $n^{\circ} \mathbf{I}$}

We present a 45-year-old male with medical history of pleural tuberculosis for which he was treated with rifampicin, isoniazid, ethambutol and pyrazinamide since 3 weeks. He was admitted to the general surgery department for a small bowel obstruction with abdominal pain, vomiting and abdominal distension.

Abdominal examination revealed a diffuse distension with loud borborygmi and a tender left flank. The rectum was empty. Abdominal $\mathrm{x}$-ray in up-right position showed fluid levels in the bowel. Biological investigations revealed a white cell count of $12700 / \mathrm{mm} 3$.

CT scan with contrast revealed a segmental thickening of the last ileal loops, distended small bowel loops without a detectable transition point, a $2 \mathrm{~cm}$ mesenteric mass associated with many mesenteric lymph nodes and magma of ileal loops some of which were contiguous to the sigmoid colon in the pelvis (Figure 1).

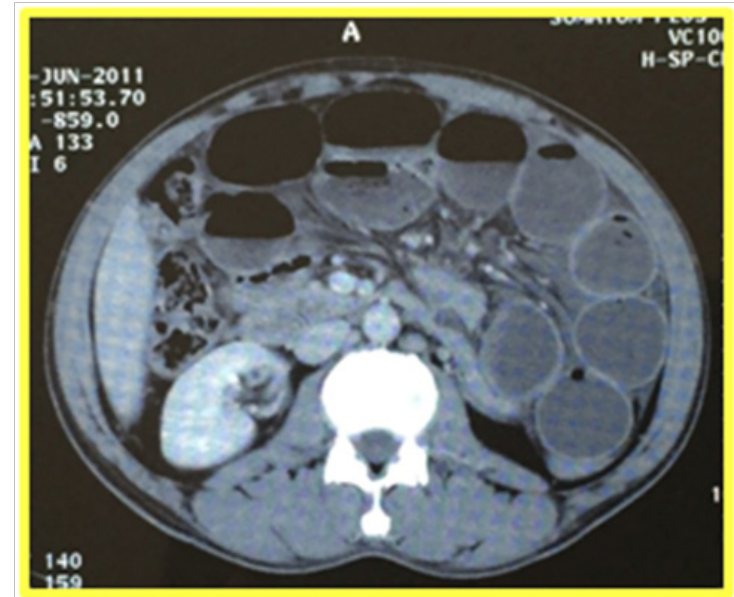

Figure I A CT scan with contrast revealed a segmental thickening of the last ileal loops and distended small bowel loops without a detectable transition point.

A naso-gastric tube was placed on suction. There was no clinical improvement and surgical intervention was immediately prepared: the
Volume 4 Issue 3 - 2016

\section{Zaafouri Haithem, Ben Bader Mourad, Khedhiri Nizar, Koubaa Wafa, Bouhafa \\ Ahmed, Ben Maamer Anis \\ Department of general surgery and department of pathological anatomy and cytology, Tunisia}

\author{
Correspondence: Zaafouri Haithem, Department of general \\ surgery and department of pathological anatomy and cytology, \\ Habib Thameur hospital, 8 Ali Ben Ayed Street's, Monftfleury \\ 1008, Tunis, Tunisia, Tel 21697348839 , \\ Email zaafouri.haithem@hotmail.fr
}

Received: December 15, 2015 | Published: March 23, 2016

bowel obstruction was related to a mesenteric mass at which the small bowel and sigmoid colon were clustered and contiguous with dilation of the small bowel upstream. We performed a resection of the small bowel and sigmoid colon with the mesenteric mass. Pathological examination of the surgical specimen found a colonic tuberculosis fistulized in the mesentery (Figure 2).

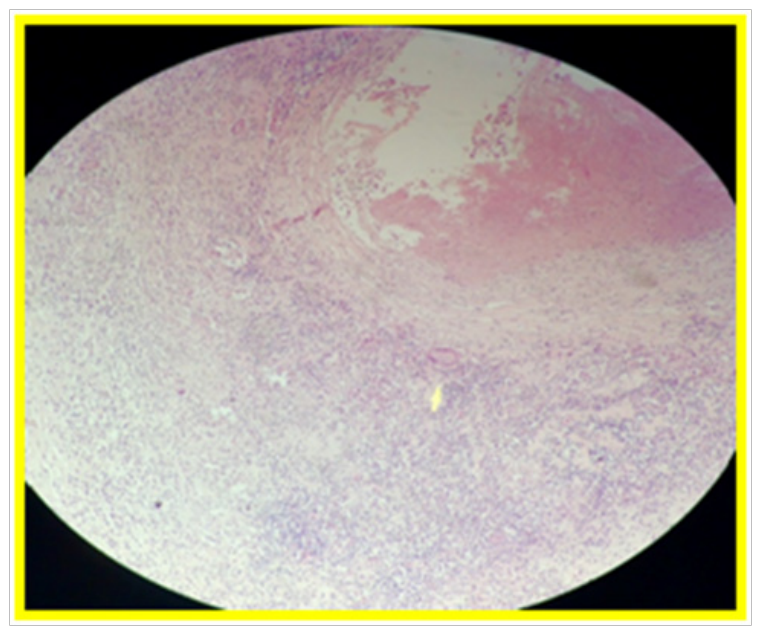

Figure 2 Histopathologic image of an epithelioid granulomas with central necrosis at colon.

He had an uneventful postoperative recovery and was discharged home with anti-TB medications. He remains asymptomatic 6 months following treatment with resolution of his symptoms.

\section{Case $n^{\circ} 2$}

A 21-year-old female was operated for an acute bowel obstruction related to a stenosis of the last ileal loop for which she had an ileocecal resection. Histological examination confirmed the diagnosis of ileo-cecal tuberculosis. She had a satisfactory evolution and was discharged. At 1 year follow-up she is doing well and has finished his course of anti-TB treatment. 


\section{Case $n^{\circ} 3$}

An 18-year-old female presented with an intermittent diarrhea associated with peripheral and profound polyadenopathy. She had a colonoscopy with colonic and ileal biopsies. The mucosa of the left colon showed a pseudo-nodular appearance and was widely ulcerated in places. Anatomopathological examination found colonic tuberculosis. A treatment with anti-TB drugs was initiated and the outcome was uneventful. ${ }^{1}$

\section{Conclusion}

Gastrointestinal tuberculosis is a quite common disease in our country, despite its prevention. Clinicians need to have gastrointestinal tuberculosis in their top diagnosis when dealing with patients with chronic gastrointestinal symptoms who are HIV-positive or coming from countries of high prevalence of TB. The signs and symptoms of intestinal TB are usually non-specific and diagnostic tests are sometime non-sensitive. A high level of evidence is required for an adequate diagnosis. Conservative management is successful in the majority of cases.

\section{Acknowledgments}

None.

\section{Funding}

None.

\section{Conflicts of interset}

The authors declare that there is no conflict of interest.

\section{References}

1. Donoghue HD, Holton J. Intestinal tuberculosis. Curr Opin Infect Dis. 2009;22(5):490-496. 